\title{
EVALUATION OF AGREEMENT BETWEEN HISTOLOGICAL CLASS CLINICAL INFERENCE OF LUPUS NEPHRITIS BASED ON CLINICAL AND LABORATORY DATA AND KIDNEY BIOPSY
}

Antonio Silaide De Araujo Junior ${ }^{1, *}$, Emília Inoue Sato, Gianna Mastroianni Kirsztajn', Ricardo de Castro Cintra Sesso ${ }^{1}$, Martin $^{1}$ Fabio Jennings Simoes ${ }^{1}$, Alexandre Wagner Silva de Souza', Edgard Torres Dos Reis Neto

1. Universidade Federal de São Paulo, São Paulo (SP), Brazil.

${ }^{\star}$ Corresponding author: juniorantoniosilaide@gmail.com

\section{BACKGROUND}

Systemic lupus erythematosus (SLE) is a multisystemic autoimmune disease and lupus nephritis (LN) is one of the main causes of morbidity and mortality in these patients. Kidney biopsy is considered the gold standard for its diagnosis and is useful for guiding therapy. The objectives were to evaluate the correlation of histological class inference based only on clinical manifestations and laboratory tests among rheumatologists and nephrologists compared to that finding in the kidney biopsy of SLE patients with LN.

\section{MATERIALS AND METHODS}

A retrospective study based on analysis of medical records of 80 SLE patients who were underwent kidney biopsy between 2010 and 2017. Two rheumatologists and two nephrologists were invited (one of each recognized and experienced specialist in the treatment of SLE patients and the other with general practice in each specialty) and received clinical and laboratory data in the form of cases, then answer questions about which histological class was expected in the kidney biopsy and which treatment would be indicated. Statistical analysis: Kappa agreement coefficient was applied to assess the agreement between evaluators and the outcome of kidney biopsy and treatment, $p=0.05$ was considered significant.

\section{RESULTS}

The average age was $33.0 \pm 10.3$ years and the mean disease duration was $11.5 \pm 6.7$ years. The mean serum creatinine and 24-hour proteinuria were, respectively, $1.85 \pm 1.88 \mathrm{mg} / \mathrm{dL}$ and $4.40 \pm 3.69 \mathrm{~g}$. Proliferative histological classes (III or IV) (61.3\%) and treatment with methylprednisolone and cyclophosphamide (62.5\%) were the most observed in the medical records. The level of agreement between the evaluators and the kidney biopsy was considered poor ( $g$ lobal Kappa $=0.364 \pm 0.029 ; p=0.001$ ) and the best result was found in the rheumatologist with experience in the treatment of SLE patients (Kappa $=0.383 \pm 0.067$; $p=0.001$ ) with a worse result in the rheumatologists with general practice in each specialty (Kappa $=0.275$ ) and both nephrologists $(K a p p a=0.316)$. Regarding the treatment, we also found a weak level of agreement (global Kappa $=0.265 \pm 0.037 ; p=0.001$ ).

\section{CONCLUSIONS}

Both rheumatologist and nephrologist with experience in the treatment of SLE patients, as well as those with general practice in their respective specialties presented a weak correlation index with kidney biopsy and treatment. Thus, kidney biopsy is a procedure that adds very relevant information to the diagnosis and treatment of patients with $L N$. 